\title{
Herramientas web 3.0 y el desempeño académico de estudiantes universitarios: estudio de estudio carrera de educación básica
}

\author{
Web 3.0 tools and the academic performance of university \\ students: case study basic education career
}

1 María Cristina Páez-Quinde

https://orcid.org/0000-0002-0654-0177

Universidad Técnica de Ambato, Facultad de Ciencias Humanas y de la Educación, Carrera de Educación Básica. Ambato, Ecuador, Grupos de investigación Research in Language and Education y Marketing C.S.

mc.paez@uta.edu.ec

2 Sonia Paola Armas-Arias

https://orcid.org/0000-0001-6923-1374

Universidad Técnica de Ambato, Facultad de Ciencias Humanas y de la Educación, Universidad Técnica de Ambato, Ambato, Ecuador, Grupo de investigación Marketing C.S

sp.armas@uta.edu.ec

3 Darwin Patricio Miranda-Ramos (iD) https://orcid.org/0000-0001-5611-9535 Universidad Técnica de Ambato, Facultad de Ciencias Humanas y de la Educación, Carrera de Educación Básica. Ambato, Ecuador dp.miranda@uta.edu.ec

4 Héctor Daniel Morocho-Lara (iD) https://orcid.org/0000-0003-3107-6238. Universidad Técnica de Ambato, Facultad de Ciencias Humanas y de la Educación, Carrera de Educación Básica. Ambato, Ecuador hd.morocho@uta.edu.ec

\begin{tabular}{r}
\hline Artículo de Investigación Científica y Tecnológica \\
Enviado: $16 / 12 / 2021$ \\
Revisado: $28 / 12 / 2021$ \\
Aceptado: $12 / 01 / 2022$ \\
Publicado:08/03/2023 \\
DOI: $\underline{\text { https://doi.org/10.33262/concienciadigital.v6i1.4.1984 }}$
\end{tabular}

Cítese: BY NC SA
Páez Quinde, M. C., Armas Arias, S. P., Miranda Ramos, D. P., \& Morocho Lara, H. D. (2023). Herramientas web 3.0 y el desempeño académico de estudiantes universitarios: estudio de estudio carrera de educación básica. ConcienciaDigital, 6(1.4), 36-49. https://doi.org/10.33262/concienciadigital.v6i1.4.1984

CONCIENCIA DIGITAL, es una Revista Multidisciplinar, Trimestral, que se publicará en soporte electrónico tiene como misión contribuir a la formación de profesionales competentes con visión humanística y crítica que sean capaces de exponer sus resultados investigativos y científicos en la misma medida que se promueva mediante su intervención cambios positivos en la sociedad. https://concienciadigital.org

La revista es editada por la Editorial Ciencia Digital (Editorial de prestigio registrada en la Cámara Ecuatoriana de Libro con No de Afiliación 663) www.celibro.org.ec

Esta revista está protegida bajo una licencia Creative Commons Attribution Non Commercial No Derivatives 4.0 International. Copia de la licencia: http://creativecommons.org/licenses/by-nc-nd/4.0/ 


\section{Palabras}

claves: TIC,

herramientas

web

3.0 ,

recursos

tecnológicos, educación, pedagogía, rendimiento académico.

\section{Keywords:}

ICT, web 3.0 tools, technological resources, education, pedagogy,

\section{Resumen}

Esta investigación se basa en la identificación de los recursos web 3.0 en la aplicación del aula de clase mediante una educación virtual y a la vez comprobar el nivel de desempeño académico que tienen los estudiantes universitarios. El objetivo de la investigación es identificar el uso de los recursos de la web 3.0 y el aporte dentro del desempeño académico de los estudiantes universitarios. La metodología utilizada es de tipo experimental-analítica mediante un enfoque por partes tomando primero lo cualitativo mediante un análisis diagnóstico enfocado en las percepciones del entorno y luego por medio de la tabulación de la data obtenida mediante una encuesta realizada a los estudiantes de la Carrera de Educación Básica, el instrumento utilizada se basa en un cuestionario estructurado de 24 ítems validado mediante el estadístico Alfa de Cronbach con un valor de 0.841 confiable para la investigación; para la comprobación de la hipótesis se utiliza el estadístico de Kolmogórov- Smirnov para k muestras independientes en el cual se seleccionan dos preguntas, la más representativas de la experimentación. Para el desarrollo de los recursos 3.0 se considera la aplicación de la metodología ADDIE la misma que permite el desempeño pedagógico y tecnológico y además del Modelo TAM, en el cual se mide la aceptación de la tecnología para este caso el de los recursos basados en la web 3.0. Los resultados de la investigación fueron altamente favorables, éstos permitieron identificar el tipo de recursos 3.0 que se pueden aplicar en una clase virtual y luego de la experimentación la aceptabilidad que éstos tienen en la población de estudio. Finalmente, se concluye que este tipo de recursos son de gran aporte para el desarrollo de una clase virtual, los mismo promueven un aprendizaje sincrónico como asincrónico, así como individual y colaborativo.

\section{Abstract}

This research is based on the identification of web 3.0 resources in the classroom application through a virtual education and once checking the level of academic performance of university students. The objective of the research is to identify the use of web 3.0 resources and the report within the academic performance of university students. The methodology used is of an experimentalanalytical type through a part-by-part approach, taking first the qualitative through a diagnostic analysis focused on the perceptions 
academic

performance. of the environment and then through the tabulation of the data obtained through a survey carried out to the students of the Career of Basic Education, the instrument used is based on a 24-item structured questionnaire validated by Cronbach's Alpha statistic with a value of 0.841 reliable for the investigation. To test the hypothesis, the Kolmogórov-Smirnov statistic is used for $\mathrm{k}$ independent samples in which two questions are selected, the most representative of the experimentation. For the development of the 3.0 resources, the application of the ADDIE methodology is considered, which allows the pedagogical and technological performance and in addition to the TAM Model, in which the acceptance of the technology is measured for this case that of the based resources on web 3.0. The results of the research were highly favorable, which allowed us to identify the type of 3.0 resources that can be applied in a virtual classroom and after experimentation the acceptability they have in the study population. Finally, it is concluded that these types of resources are of great contribution to the development of a virtual classroom, they promote synchronous and asynchronous learning as well as individual and collaborative.

\section{Introducción}

Debido a la pandemia COVID 19, la educación presencial se vio afectada y tomo un giro drástico, educación virtual. Considerando que no es un tema nuevo la implantación de las Tac y Tic en el proceso educativo, sigue siendo un tema poco explorado en nuestro contexto. Aun se considera permitente la enseñanza tradicional, que no implementa tantos recursos tecnológicos, puesto la carencia de infraestructura y materiales en muchas ocasiones se hace evidente, ya sea en el sector urbano o rural. Ahora bien, la educación desde marzo 2020 ha de cambiar, creando la oportunidad de trabajar en un entorno virtual que, aunque genero diversos inconvenientes en su principio, también ha brindado la posibilidad de una transformación docente y su capacitación tecnológica.

Vilatuña (2020), en su tesis de maestría denominada "Sitio web 3.0 de escritura creativa para estudiantes de tercer año de educación general básica", buscó elaborar un sitio web 3.0 para contribuir al proceso de enseñanza aprendizaje de escritura creativa de los estudiantes de tercer año. Se recurre al empleo del método empírico con la técnica de la observación dirigido a los estudiantes para el diagnóstico en la adquisición de destrezas necesarias en la escritura creativa, Además se utiliza el método teórico a través de encuestas a los docentes de Lengua y Literatura sobre el uso de TIC en el proceso de enseñanza. Con esto se llega a la conclusión, que el sitio web interactivo 3.0 
denominado JIMDO, es de gran utilidad al facilitar la enseñanza y aprendizaje de la escritura creativa, al desarrollarse la percepción y la memoria como habilidades cognitivas, a su vez de favorecer a las habilidades afectivas y sociales.

Salcedo et al. (2020), en su artículo "La importancia de la web 3.0 y 2.0 en el desarrollo de la pedagogía educativa en tiempos de pandemia”, como artículo de revisión, busca dar respuesta sobre sí los docentes están preparados para hacer frente a una educación en tiempos de pandemia del Covid19. Es así como las herramientas tecnológicas que se han empleado para mantener en marcha el sistema educativo una vez que la pandemia se ha suscitado. Se han convertido en una necesidad de realizar orientaciones pedagógicas, técnicas de enseñanza, videos tutoriales, aulas virtuales en base a la web 2.0 y 3.0 , vista no solo como un elemento de apoyo sino como una forma de transformarla en una educación.

Con esto concluyen que aún falta por profundizar esta temática, las experiencias actuales servirán a futuros investigadores en el desarrollo de redes y web que aporten a la pedagogía post pandemia.

Guix (2021), en su artículo investigativo con el tema "Aplicaciones web 3.0 para la mejora del aprendizaje. Una experiencia con profesores de educación secundaria obligatoria". Se presenta un marco conceptual sobre recursos en los que se combina pedagogía y tecnología. Es un estudio empírico con una metodología mixta, se trata de un estudio de caso de tres instituciones de educación superior. La información es recabada a través de cuestionarios elaborados por grupos de discusión. Aquí se constata que existen escasas empresas dedicadas a desarrollar aplicaciones 3.0 enfocadas en este nivel. Además, se destaca la distracción de los estudiantes y un bajo nivel de competencias en el profesorado en el manejo de las TIC. Al finalizar la investigación afirman que existe una escasa oferta de tecnología 3.0 orientada a la educación secundaria, por lo que los docentes no logran disponer totalmente de herramientas que puedan ser empleados como recursos en las aulas. En cuanto al aprendizaje los docentes reconocen que la tecnología 3.0 ayuda en la autonomía, iniciativa, trabajo colaborativo y motivación en el proceso educativo.

Web 3.0

La web 3.0 o también denominada la web semántica para Lima-Montenegro \& FernandezNodarse (2017), afirma que parte de añadir información para completar contenido incrementando el significado de datos para lograr un mayor intercambio de información incluso en la manera en cómo las personas se relacionan socialmente y se comunican. Corino (2017), compara la web 2.0 y la web 3.0, en la menciona que la web 2.0 es una herramienta que se gestiona por el ser humano, mientras que la web $3.0 \mathrm{o}$ la web semántica se orienta a protagonizar los procesadores de información que pueden 
ser ejecutados desde cualquier dispositivo.

En la educación es necesaria la organización y personalización de la información por lo que las herramientas Web dan un alto grado para poder organizarlas y personalizarlas. Llorente (2012), muestra a la Web 3.0 como aquella que incluye contenidos semánticos a aquellos documentos que la integran. Su ejecución se realiza por las máquinas y se basa en la información importante que es descubierta en perfiles de la red.

Niño et al. (2019), manifiestan que la Web 3.0 brinda herramientas en un escenario dinámico que contribuyen al estudiante en el proceso de enseñanza aprendizaje. Puesto que no solo espera que la información llegue, sino que además tiene la posibilidad de ir por ella, se puede buscarla, compartirla e incluso crearla para lograr así una retroalimentación.

\section{Características Web 3.0}

La situación que generó la aparición de una nueva etapa de la web recae en la necesidad de contar con un Internet cada vez más inteligente, en la que los usuarios puedan realizar

búsquedas que estén más cercanas a un lenguaje natural. Por lo que las búsquedas arrojen información más relevante de acuerdo con el significado del contenido web. Dentro de las principales características de la Web 3.0 se encuentran en la Figura 1:

\section{Figura 1}

Web 3.0 características

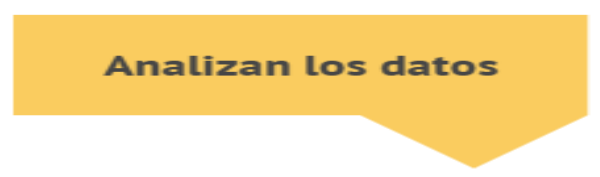

Asistencia de inteligencia artificial y semántica

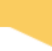

\section{Registra los historiales de los usuarios}

Tecnologías del aprendizaje y conocimiento

Los sistemas educativos se están trasformando, por ende, los modos y las metodologías de enseñanza están cambiando. Con esto surge la posibilidad de completar el proceso 
de enseñanza aprendizaje con el empleo de la tecnología que requiere de la necesidad de adquirir competencias digitales para alfabetizar y a su vez aprender a usar las TIC para luego transfórmalas en las TAC y potenciar en los estudiantes el desarrollo de habilidades sociales. Esto involucra a las instituciones y a los docentes a educar dando un paso del individualismo a buscar un beneficio para todos (Santos et al., 2016).

En el contexto educativo las TAC conocidas como las Tecnologías del Aprendizaje y el Conocimiento buscan redireccionar las TIC hacia un empleo más pedagógico y formativo, es así como TAC (Priyaadharshini et al., 2020), permiten explorar herramientas tecnológicas en favor del aprendizaje para adquirir conocimientos, puesto que van más allá de aprender a usar las TIC. Con esto se buscar una actualización constante de los aprendizajes con una generación de entornos virtuales que se pueden emplear en el aula como en otros contextos donde se busca una mayor participación y autonomía el estudiante (Velasco, 2017).

El rendimiento académico recae en lo que son las calificaciones cuantitativas que obtiene un estudiante; sin embargo, Zamora et al. (2015), concuerda en que no solo se debe analizar dichos resultados numéricos para catalogar a un estudiante como bueno, regular o deficiente, puesto que son varios los factores que pueden incidir en resultados cuantitativos bajos como lo muestra la figura 2. En la actualidad el desempeño académico tomas un concepto más acorde a la productividad del estudiante, el cumplimiento de objetivos educativos y la calidad del resultado obtenido del desarrollo de actividades en clase y en casa (Assuad et al., 2020).

\section{Figura 2}

Factores exógenos y endógenos

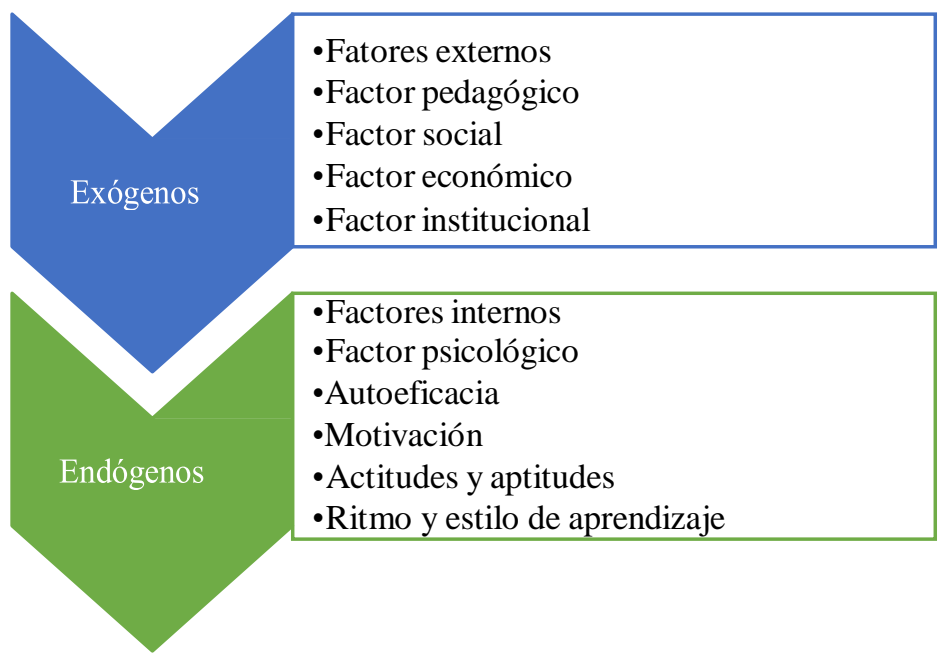

Entre los factores que inciden en el desempeño académico están los factores exógenos y endógenos, los cuales expresan como tanto factores externos como internos tienen una relación directa con el desempeño académico de los estudiantes. Es decir, la 
situación familiar, social, económica, va a inferir en los resultados que obtenga el discente en su proceso de aprendizaje. He ahí la importancia de no solo considerar las evaluaciones sumativas, ya que pueden generar resultados erróneos al momento de evaluar los conocimientos. Así también, el factor psicología, actitudes y aptitudes del estudiante pueden redimir en que su desempeño académico no sea el mejor en cuanto a puntuación, ya que esto va de mano con los ritmos y estilos de aprendizaje (Merkulova et al., 2019).

Otro factor que influye en el desempeño académico es la importancia de las expectativas familiares, es decir, la familia juega un rol fundamental en aquello. El nivel socioeconómico y cultural, hábitos de lectura, tendrán mucho impacto en los estudiantes, al momento de aprender en base a la observación, si el niño no percibe que en su eterno la lectura no comprende mayor importancia, será difícil que en él se genere un hábito de lectura. Al contrario de hogares en los cuales se fomente la lectura, participación en actividades recreativas, organicen noches familiares de juegos como ajedrez, sudoku, sopa de letras, entre otros (Guntha et al., 2020).

La motivación, los estilos, ritmos y hábitos de estudio, autorregulación y la decisión vocacional, también se consideran como factores que inciden en el desempeño académico de acuerdo con lo que se muestra en la figura 3.

\section{Figura 3}

\section{Motivación, estilos, ritmos y hábitos de estudio}

\begin{tabular}{|c|c|}
\hline Motivación & $\begin{array}{l}\text {-Impulso que genera acciones en las personas. } \\
\text { - Fuerza interna del individuo }\end{array}$ \\
\hline Estilos & $\begin{array}{l}\text {-Visual } \\
\text {-Auditivo } \\
\text {-Verbal } \\
\text {-Kinestésico }\end{array}$ \\
\hline Ritmos & $\begin{array}{l}\text {-Rápido - moderado- lento } \\
\text {-Es la capacidad con la que aprende el individuo en un determinado tiempo }\end{array}$ \\
\hline Hábitos de estudio & $\begin{array}{l}\text {-Establecer horarios } \\
\text { - Lugar tranquilo para estudiar } \\
\text {-Evitar distracciones }\end{array}$ \\
\hline Autorrequlación & $\begin{array}{l}\text { - Autoconocimiento } \\
\text { - Control de emociones } \\
\text { - Eauilibrio en obliaaciones v tiemno propio }\end{array}$ \\
\hline Decisión vocacional & $\begin{array}{l}\text { - Elecciones a futuro para una profesión o un oficio } \\
\text { - Predilección a una carrera en específico } \\
\text { - Destrezas, habilidades, capacidades }\end{array}$ \\
\hline
\end{tabular}

Además, Álvarez-Dardet et al. (2020) explica que "el factor práctico de aula está directamente relacionado con el rendimiento académico de los estudiantes". 
Esto bien, se entiende en que el trabajo dentro del aula sea este mucho o poco, va a incidir de manera directa en el desempeño académico de los estudiantes. Se creía que la repetición era la forma más eficaz de que un estudiante aprenda, pero, ahora el docente comprende que el aprendizaje debe ser significativo, innovador y de utilidad.

\section{Metodología}

La investigación fue de tipo experimental-analítica en donde se realizó el uso de la herramienta Jamboard como experimentación dentro del desarrollo de la clase; y de carácter analítico puesto que en base de los resultados obtenidos mediante la encuesta se pudo tomar decisiones en primer lugar con el uso de la herramienta web 3.0 apropiada y luego la aceptabilidad de ésta en la población de estudio. El enfoque aplicado es por partes, dentro de lo cualitativo se midieron percepciones mediante la observación y el diagnóstico realizado con los estudiantes de la carrera de educación básica; en la parte cuantitativa se procedió sacar tendencias de acuerdo a la data tabulada mediante el cuestionario estructurado en el cual se midieron aspectos como frecuencia de uso de herramientas colaborativas web 3.0, estrategias utilizadas por parte del docente en educación virtual, herramientas colaborativas enfocadas a la retroalimentación, evaluación y desarrollo de la clase. Para el uso del instrumento se procedió con la validación mediante el estadístico Alfa de Cronbach obteniendo como resultado 0.841 teniendo confiabilidad de los ítems dentro de la investigación.

La experimentación se desarrolló por el lapso de 15 semanas con estudiantes de primero, segundo y octavo semestre en las asignaturas de TIC, Convergencia de Medios Educativos y Taller de Escritura del Informe de Investigación; la metodología utilizada para el desarrollo de las clases mediante Jamboard se basó en ADDIE figura 4, la cual se enfoca en la enseñanza por medio de la tecnología, considerada como un estándar en desarrollo de recursos, aplicaciones o software educativos tanto de forma presencial como virtual.

Figura 4

ADDIE-Metodología

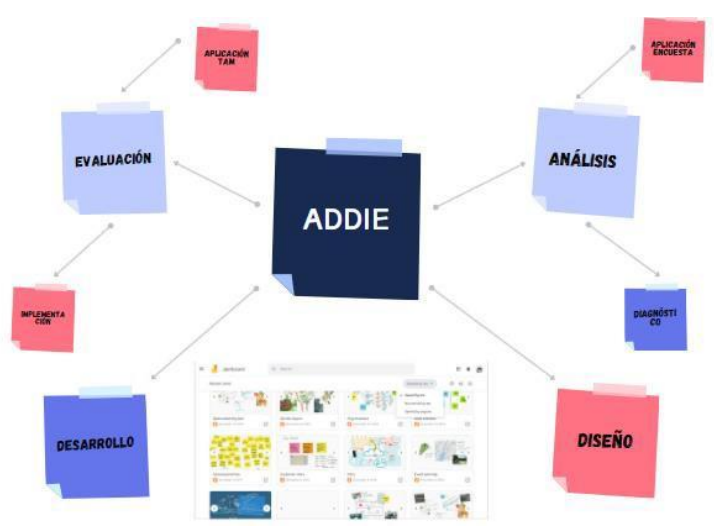


La experimentación desarrollada fue en un período de 15 semanas en donde los estudiantes pudieron desarrollar actividades grupales, individuales de forma sincrónica y asincrónica, así también como el desarrollo de la clase dentro de la educación virtual; los parámetros que se tomaron en consideración para verificar la mejora en el desempeño académico fueron la participación en clase, manejo de referentes bibliográficos y el uso de la herramienta en el trabajo colaborativo.

\section{Resultados}

Los resultados que se muestran a continuación se basan en las preguntas más representativas seleccionadas dentro de la experimentación.

De esta forma es cómo se realiza el cálculo del estadístico de Kolmogórov-Smirnov en donde se pudo comprobar la hipótesis tabla 1.

Tabla 1

Herramientas web 3.0 para aprender

\begin{tabular}{|c|c|c|c|}
\hline & Ítems & Frecuencia & Porcentaje \\
\hline \multirow{5}{*}{ Válidos } & Kahoot & 31 & 34,4 \\
\hline & Wix & 1 & 1,1 \\
\hline & Canva & 30 & 33,3 \\
\hline & $\begin{array}{l}\text { Redes sociales (Facebook, } \\
\text { Instagram, Tik-Tok) }\end{array}$ & 3 & 3,3 \\
\hline & $\begin{array}{l}\text { Página personal (Blog, } \\
\text { correo electrónico) }\end{array}$ & 3 & 3,3 \\
\hline \multirow{4}{*}{ Válidos } & $\begin{array}{l}\text { Plataformas educativas } \\
\text { (Moodle, easle) }\end{array}$ & 7 & 7,8 \\
\hline & $\begin{array}{c}\text { Dispositivos móviles } \\
\text { (WhatsApp, Telegram, } \\
\text { viber, etc.) }\end{array}$ & 1 & 1,1 \\
\hline & Zoom, Teams & 14 & 15,6 \\
\hline & Total & 90 & 100,0 \\
\hline
\end{tabular}

Una de las herramientas más utilizada dentro del proceso de aprendizaje por parte de los estudiantes de Kahoot, con un 34.44\%; seguida de Canva con un 33.33\%; algunos estudiantes utilizan herramientas de comunicación sincrónica como Zoom y Teams en un $15.56 \%$; por otro lado $7.78 \%$ de estudiantes utilizan la plataforma educativa como medio tecnológico de aprendizaje dentro de clases virtuales; en lo particular la mayoría de estudiantes prefieren hacer uso de herramientas de gamificación basadas en actividades de competencia como lo es Kahoot.

En la tabla 2 se puede evidenciar la frecuencia de uso de las herramientas web 3.0 enfocadas en la educación virtual de los estudiantes. 


\section{Tabla 2}

Frecuencia de uso de las herramientas web 3.0

\begin{tabular}{cccc}
\hline & Ítems & Frecuencia & Porcentaje \\
\cline { 2 - 4 } & Nunca & 2 & 2,2 \\
Raramente & 5 & 5,6 \\
Válidos & Ocasionalmente & 17 & 18,9 \\
& Frecuentemente & 56 & 62,2 \\
& Muy & 10 & 11,1 \\
& frecuentemente & & 100,0 \\
\hline
\end{tabular}

De un total de 90 estudiantes que fueron evaluados, el $62.22 \%$ afirma que utiliza herramientas web 3.0 dentro del trabajo colaborativo para mejorar su rendimiento académico, el 18,89\% ocupa estas herramientas de manera ocasional, el $11.11 \%$ utiliza las herramientas de forma muy frecuente; existen porcentajes mínimos como el $5.56 \%$ y el $2.22 \%$ las ocupan de forma rara y nunca respectivamente. Con esto se puede concluir que este tipo de herramientas promueven el trabajo en equipo y a la vez les permite mejorar el rendimiento académico porque puede hacer una participación de forma sincrónica basado en ellos contenidos que se desarrollan en clases virtuales.

Haciendo referencia a la tabla 3, se puede evidenciar las herramientas más utilizadas para la consolidación de conocimiento dentro de la

Tabla 3

Herramientas para consolidar conocimiento

\begin{tabular}{cccc}
\hline & Ítems & Frecuencia & Porcentaje \\
\cline { 2 - 4 } & Moodle & 32 & 35,6 \\
Kahoot & 22 & 24,4 \\
Válidos & Redes sociales & 8 & 8,9 \\
& Entornos Virtuales & 26 & 28,9 \\
Inmersivos & & 2,2 \\
Contenidos 3D & 2 & 100,0
\end{tabular}

De 90 estudiantes que participaron en la experimentación 32 de ellos que representan el $35.56 \%$ utilizan la plataforma Moodle para consolidar conocimiento, tomando en cuenta que esta plataforma es de aprendizaje asincrónico, muy utilizado en educación 
virtual; 26 estudiantes que representa el $28.89 \%$ de la población usan entornos virtuales inmersivos como classdojo, pixtón y toondoo; 22 estudiantes que marcan una tendencia del $24.42 \%$ utilizan kahoot como herramienta para consolidar conocimiento y de esta forma mejorar su rendimiento académico.

Para la comprobación de la hipótesis en donde se afirma que los recursos web 3.0 mejoran el desempeño académico en los estudiantes universitarios al tener un $\mathrm{P}$ (valor) menor a 0.05 se rechaza la hipótesis nula y se comprueba la hipótesis establecida.

Al utiliza el estadístico de prueba de Kolmogórov-Smirnov se comprueba que las variables utilizadas para la investigación tienen un nivel de concordancia entre la data obtenida y la distribución teórica especificada evidenciado en la tabla 4.

Tabla 4

Test de Kolmogórov-Smirnov

\begin{tabular}{|c|c|c|c|c|}
\hline & & $\begin{array}{c}\text { Tipos de } \\
\text { herramientas } \\
3.0 \text { que usted } \\
\text { utiliza para } \\
\text { aprender: }\end{array}$ & $\begin{array}{l}\text { Frecuencia del } \\
\text { trabajo } \\
\text { colaborativo } \\
\text { mediante uso de } \\
\text { herramientas web }\end{array}$ & $\begin{array}{l}\text { Herramienta } \\
\text { consolidar } \\
\text { conocimiento }\end{array}$ \\
\hline $\mathrm{N}$ & & 90 & 30 & 90 \\
\hline \multirow[t]{2}{*}{ Normal Parameters $a, b$} & Mean & 4,08 & 3,74 & 2,38 \\
\hline & $\begin{array}{l}\text { Std. } \\
\text { Deviation }\end{array}$ & 3,339 & ,815 & 1,295 \\
\hline \multirow{3}{*}{$\begin{array}{l}\text { Most Extreme } \\
\text { Differences }\end{array}$} & Absolute & 315 & ,356 & ,215 \\
\hline & Positive & 315 & ,266 & ,215 \\
\hline & Negative &,- 178 &,- 356 &,- 206 \\
\hline Kolmogorov-Smirnov Z & & 2,993 & 3,381 & 2,038 \\
\hline Asymp. Sig. (2-tailed) & & ,000 & 000 & 000 \\
\hline
\end{tabular}

a. Test distribution is Normal.

b. Calculated from data.

\section{Conclusiones}

- Los recursos web 3.0 en la actualidad han permitido una mejora en el desempeño de los estudiantes, tomando en consideración aspectos como: un uso correcto de este tipo de recursos, su frecuencia de uso y la forma en cómo se los debe implementar en el aula. La utilización de este tipo de recursos le involucra de forma directa al docente, puesto que es él quien debe adaptar sus clases basados 
en metodologías apropiadas y recursos que los estudiantes participen de forma activa.

- Las tecnologías del aprendizaje y del conocimiento proporcionan un aprendizaje significativo, en donde el rol del estudiante cumple un papel importante, deja de ser el ente pasivo en el proceso educativo y empieza a generar conocimiento adaptado a las necesidades de la educación virtual, todo esto basado en una educación virtual y el uso correcto de las herramientas web 3.0; en el caso específico de Jamboard en donde los estudiantes aprenden basados en diferentes interacciones que tienen con sus equipos de trabajo y con su contexto.

\section{Agradecimiento}

Un agradecimiento a la Universidad Técnica de Ambato, a la Dirección de Investigación y Desarrollo (DIDE) por apoyar el proyecto de investigación Desarrollo de herramientas web

3.0 en la educación como aporte en el aprendizaje colaborativo SFFCHE5 y ser parte de los grupos de investigación: Research in Language and Education y Marketing C.S.

\section{Referencias bibliográficas}

Álvarez-Dardet, S., Lara, B., \& Pérez-Padilla, J. (2020). Older adults and ICT adoption: Analysis of the use and attitudes toward computers in elderly Spanish people. Computers in Behavior, 110, $106377 . \quad$ Doi: https://doi.org/10.1016/j.chb.2020.106377

Assuad, C., Tvenge, N., \& Martinsen, K. (2020). System dynamics modelling and learning factories for manufacturing systems education. Procedia CIRP, 88, 1518. Doi: https://doi.org/10.1016/j.procir.2020.05.003

Corino, C. (2017). Evolución de la web 2.0 a la 3.0, y su impacto en la empresa. (Tesis de maestría) Universidad de Cantabria.

Guix Parés, E. (2021). Aplicaciones web 3.0 para la mejora del aprendizaje. Didáctica, innovación $\quad y \quad$ multimedia, https://raco.cat/index.php/DIM/article/view/388775/482239

Guntha, R., Hariharan, B., \& Rangan, P. (2020). Analysis of Multimedia Communication Issues in the Immersive Smart Classroom System - A Control Center Approach. Procedia Computer Science, 117, 2600-2609. Doi: https://doi.org/10.1016/j.procs.2020.04.282 
Lima-Montenegro, S., \& Fernandez-Nodarse, F. (2017). La educación a distancia en entornos virtuales de enseñanza aprendizaje. Reflexiones didácticas. Atenas, 3(39), 31-47.

Llorente, M. (2012). De la web tradicional a la web semántica: cambios y aplicación al ámbito educativo. Apertura, 3(1), 120-129.

Merkulova, I., Shavetov, S., Borisov, O., \& Gromov, V. (2019). Object detection and tracking basics: Student education. IFAC-Papers Online, 52(9), 79-84. Doi: https://doi.org/10.1016/j.ifacol.2019.08.128

Niño, J., Fernández, F., \& Duarte, J. (2019). Diseño de un recurso educativo digital para fomentar el uso racional de la energía eléctrica en comunidades rurales. Saber, Ciencia y Libertad, 14(2), 256-272.

Priyaadharshini, M., Natha-Mayil, N., Dakshina, R., S., \& Bettina-Shirley, R. (2020). Learning Analytics: Game-based Learning for Programming Course in Higher Education. Procedia Computer Science, 172, 468-472. Doi: https://doi.org/10.1016/j.procs.2020.05.143

Salcedo, D., Villamar, D., \& De Rosario, E. (2020). La importancia de la web 3.0 y 2.0 en el desarrollo de la pedagogía educativa en tiempos de pandemia. Reciamuc, 14(2), 13-23. https://revista-cientificainternacional.org/index.php/revista/article/view/8/35

Santos, A., Carreño, J., \& Camargo, C. (2016). Modelo Espiral de Competencias Docentes TICTACTEP aplicado al Desarrollo de Competencias Digitale, 19, 3948.

Velasco, M. (2017). Las TAC y los recursos para generar aprendizaje. Infancia Educación y Aprendizaje, 3(2), 771-777. https://doi.org/10.22370/ieya.2017.3.2.796.

Vilatuña, M. D. (2020). Sitio web 3.0 de escritura creativa para estudiantes de tercer año de Educación

General. http://repositorio.uisrael.edu.ec/bitstream/47000/2661/1/UISRAEL-EC-MASTEREDUC-378.242-2020-134.pdf

Zamora Mora, V., Soares, P., Echeverria, C., Hernández, R., \& Mijangos, C. (2015). Composite chitosan/Agarose ferrogels for potential applications in magnetic hyperthermia. Gels., 1, 69-80.

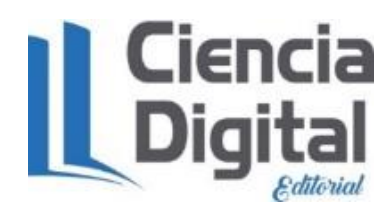




\section{DDigital}

El artículo que se publica es de exclusiva responsabilidad de los autores y no necesariamente reflejan el pensamiento de la Revista Conciencia Digital.

\section{Ciencia \\ LDigital}

El artículo queda en propiedad de la revista y, por tanto, su publicación parcial y/o total en otro medio tiene que ser autorizado por el director de la Revista Conciencia Digital.
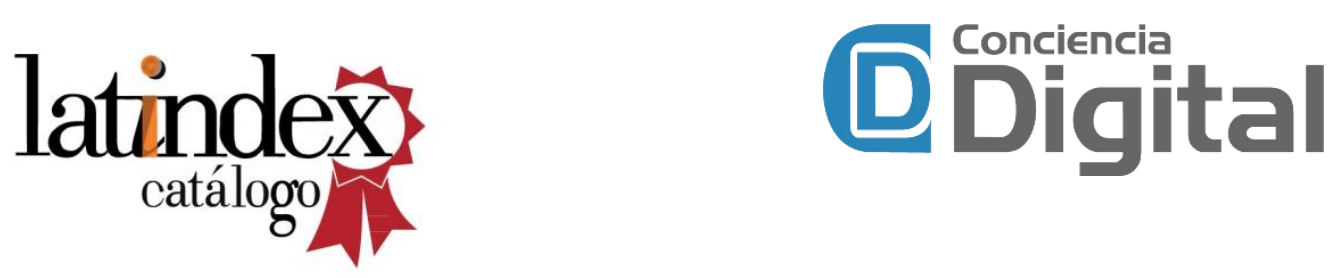

Indexaciones

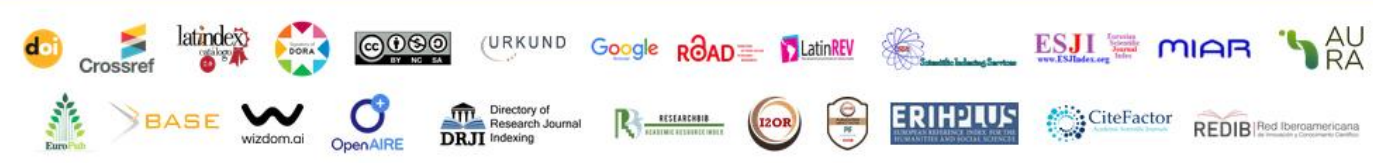

